\title{
Validation of CT Attenuation Correction for High-Speed Myocardial Perfusion Imaging Using a Novel Cadmium- Zinc-Telluride Detector Technique
}

Bernhard A. Herzog ${ }^{1}$, Ronny R. Buechel ${ }^{1}$, Lars Husmann ${ }^{1}$, Aju P. Pazhenkottil ${ }^{1}$, Irene A. Burger ${ }^{1}$, Mathias Wolfrum ${ }^{1}$, Rene N. Nkoulou ${ }^{1}$, Ines Valenta ${ }^{1}$, Jelena R. Ghadri ${ }^{1}$, Valerie Treyer ${ }^{1}$, and Philipp A. Kaufmann ${ }^{1,2}$

${ }^{1}$ Cardiac Imaging, University Hospital Zurich, Zurich, Switzerland; and ${ }^{2}$ Zurich Center for Integrative Human Physiology, University of Zurich, Zurich, Switzerland

The aim of this study was to validate attenuation correction (AC) using low-dose standard CT for myocardial perfusion imaging (MPI) on a novel ultra fast $\gamma$-camera with cadmium-zinc-telluride (CZT) detector technology. Methods: Sixty-six patients (body mass index $\pm \mathrm{SD}, 27.2 \pm 3.5 \mathrm{~kg} / \mathrm{m}^{2}$; range, $19.1-36.0 \mathrm{~kg} / \mathrm{m}^{2}$ ) underwent a 1-d ${ }^{99 m}$ Tc-tetrofosmin adenosine stress-rest imaging protocol with 15-min acquisitions on a standard dualhead SPECT camera. All scans were repeated within minutes on the CZT camera, with 3-min acquisitions for stress (low dose) and 2-min acquisitions for rest (high dose) as recently established. We compared maximum myocardial uptake (20segment model) from CZT versus standard SPECT MPI by intraclass correlation without and with CT AC. In addition, clinical agreement for each coronary territory for all scans from both devices was assessed, and Bland-Altmann (BA) limits of agreement for percentage uptake were calculated. Results: The clinical agreement between CZT and standard SPECT cameras was $96 \%$ for noncorrected low- and high-dose images $(r=0.90$ and $\mathrm{BA}=-18$ to 15 , and $r=0.91$ and $\mathrm{BA}=-15$ to 16 , respectively), and agreement after $A C$ was $96 \%$ for low- and $99 \%$ for high-dose images $(r=0.87$ and $\mathrm{BA}=-16$ to 14 , and $r=0.88$ and $\mathrm{BA}=-16$ to 14 , respectively). Conclusion: Our results support that $A C$ of MPI on the novel CZT camera, compared with AC MPI on a conventional SPECT camera, is feasible because it provides a high correlation of segmental tracer uptake and an excellent clinical agreement.

Key Words: cardiology (clinical); SPECT; attenuation correction; cadmium zinc telluride

J Nucl Med 2010; 51:1539-1544

DOI: 10.2967/jnumed.110.078170

\section{S} diagnosis and prognosis of ischemic coronary artery disease (CAD) as a guide for patient management in daily clinical routine. However, technical limitations such as soft-tissue attenuation of tracer activity mainly from the inferior myocardial wall $(1,2)$ may affect its specificity. Prone or com-

Received Apr. 13, 2010; revision accepted Jul. 8, 2010.

For correspondence or reprints contact: Philipp A. Kaufmann, Cardiac Imaging, University Hospital Zurich, Ramistrasse 100, CH-8091 Zurich, Switzerland.

E-mail: pak@usz.ch

COPYRIGHT @ 2010 by the Society of Nuclear Medicine, Inc. bined supine-prone acquisition (3) and external radionuclide sources, such as ${ }^{153} \mathrm{Gd}(4)$, have been evaluated for attenuation correction (AC). Recently, attenuation maps obtained from unenhanced CT X-ray scans have been introduced as a reliable method for $\mathrm{AC}$ of myocardial perfusion imaging (MPI) in clinical practice $(1,5,6)$. CT AC proved superior to prone MPI acquisition (7) and has several advantages over external radionuclide sources for $\mathrm{AC}$, such as higher photon flux and hence no influence by cross-talk from the SPECT radionuclide, no decay of transmission source, and shorter scan times $(8,9)$.

Not only the need for increased patient throughput to improve cost-effectiveness but also the necessity of reducing patients' discomfort and minimizing motion artifacts have induced the search for strategies for shortening MPI acquisition and protocol times. Recently, a new $\gamma$-camera was introduced with a novel generation of detectors using cadmium-zinc-telluride (CZT) semiconductor technology, which allows reduction of scan time down to a few minutes $(10,11)$. Despite these remarkable refinements, the discrimination of artifacts due to soft-tissue attenuation from real perfusion defects has remained a challenge. Therefore, the aim of this study was to validate x-ray-based CT AC of MPI from a CZT camera using a conventional dual-head SPECT camera as a standard of reference.

\section{MATERIALS AND METHODS}

The present study included 66 consecutive patients who were referred to MPI for the assessment of ischemic CAD. All patients underwent MPI on a standard dual-detector SPECT camera (Ventri; GE Healthcare) and on a novel ultrafast CZT camera (Discovery NM 530c; GE Healthcare). The study protocol was approved by the local ethics committee, and written informed consent was obtained from each patient.

\section{Study Protocol}

All 66 patients underwent a 1 -d ${ }^{99 m}$ Tc-tetrofosmin adenosine stress-rest imaging protocol (12). The patients were told to refrain from caffeine-containing beverages for at least $12 \mathrm{~h}$ before the MPI study. Pharmacologic stress was induced by infusion of adenosine at a rate of $140 \mu \mathrm{g} / \mathrm{kg} / \mathrm{min}$. A standard dose of 300$350 \mathrm{MBq}$ of ${ }^{99 \mathrm{~m}} \mathrm{Tc}$-tetrofosmin was injected $3 \mathrm{~min}$ into the pharmacologic stress. Ninety minutes later, stress study images were 
acquired on the standard SPECT camera, followed within minutes by the acquisition on the CZT camera. Thereafter, a tracer dose of 2.5-3 times the stress dose was administered at rest, followed by image acquisition on both cameras with the same sequence and protocol as at stress (Fig. 1).

\section{CT AC}

To create AC maps, all patients underwent an unenhanced 64slice CT examination on a standalone Light Speed VCT Scanner (GE Healthcare) during a breath-hold, as previously reported (13). The scans covered the entire heart using prospectively electrocardiogram-triggered sequential images at $75 \%$ of the R-R interval, 2.5-mm section thickness, and 0.35-s gantry rotation times at 120 $\mathrm{kV}$ and 200-250 mA, depending on the patient's size. For CT, attenuation-corrected images were reconstructed with $5.0-\mathrm{mm}$ thickness using a reconstruction algorithm with a $512 \times 512$ matrix and a full chest-sized adapted field of view of $50 \times 50 \mathrm{~cm}$. The reconstructed $\mathrm{CT}$ images were then transferred to the Xeleris workstation (GE Healthcare) for AC map generation (13).

\section{SPECT Myocardial Perfusion Image Acquisition and Reconstruction}

The first acquisition was performed on a standard dual-head SPECT camera with a low-energy, high-resolution collimator; a $20 \%$ symmetric window at $140 \mathrm{keV}$; a $64 \times 64$ matrix; and an elliptic orbit with step-and-shoot acquisition at $3^{\circ}$ intervals over a $180^{\circ}$ arc $\left(45^{\circ}\right.$ right anterior oblique to $45^{\circ}$ left posterior oblique) with 30 steps ( 60 views). Scan time was set to $25 \mathrm{~s}$ per frame for stress and rest. This scan time resulted in a total acquisition time of $14 \mathrm{~min} 52 \mathrm{~s}$ (including interstep rotation time) for each scan, as recommended by the American Society of Nuclear Cardiology (14). Images were reconstructed on the same dedicated workstation as was used for CT maps, with a standard iterative reconstruction algorithm-ordered-subset expectation maximization (2 iterations, 10 subsets) with and without CT AC—into standard short-axis and vertical and horizontal long-axis views and polar maps of perfusion encompassing the entire left ventricle. A Butterworth postprocessing filter (frequency, 0.50; order, 10) was applied to the reconstructed slices.

\section{CZT Image Acquisition and Reconstruction}

The second scan was acquired on a CZT camera with pinhole collimation (15), for which the conventional sodium iodide (NaI)

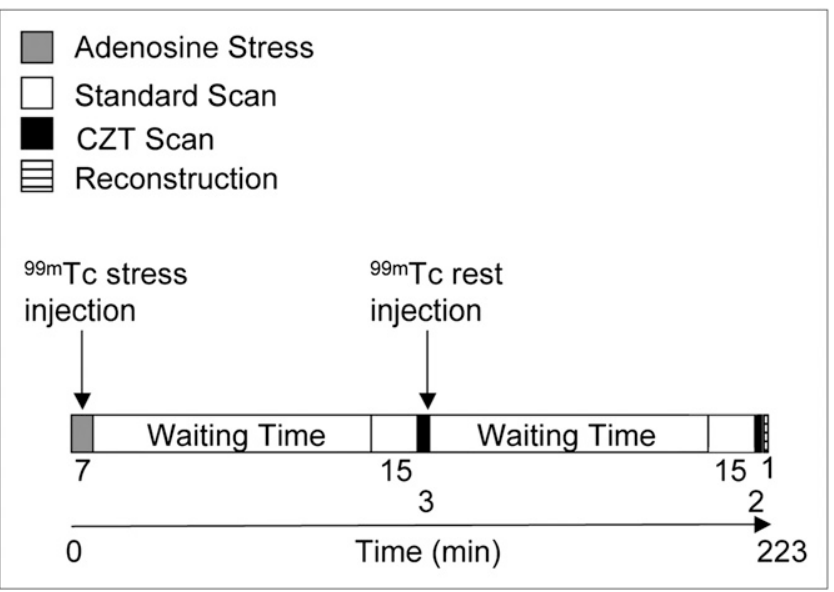

FIGURE 1. Scheme of study protocol. crystals have been replaced by CZT semiconductor detectors that directly convert radiation into electric signals without any of the steps of violet light production, transport, and conversion as occurs with $\mathrm{NaI}$ crystals (16). Compared with a dedicated cardiac $\gamma$-camera (Ventri; GE Healthcare) $(10,11)$, for the CZT camera the energy and spatial resolutions (radial resolution, $4.3 \mathrm{~mm}$ ) were improved by a factor of 2 , and the sensitivity $(21.0$ counts/s/MBq $[21.0$ counts/s/mCi]) was improved by a factor of almost 4 . The new CZT technology is extremely compact, and this miniaturizationtogether with the increased resolution-offers the opportunity to construct a stationary array of 19 small $\gamma$-cameras packed closely together and focused on the heart. The stationary array simultaneously acquires all the views necessary for tomographic reconstruction, saving the time taken by conventional cameras to acquire views while rotating around the subject. All the views are simultaneously focused on the heart to maximize efficiency of cardiac imaging. To fit the multiple views, the image is reduced by means of pinhole collimation, matching the miniaturization to the improved intrinsic pixel resolution of the detectors. This reduction allows for the maximization of detector surface use, which translates into increased system efficiency. The pinhole geometry has several advantages: the reduction in pinhole sensitivity with increasing distance significantly diminishes the contribution of background organs and tissues to the cardiac data, facilitating reliable 3-dimensional iterative reconstruction. In addition, the oblique angles of incidence also improve the already superior energy resolution of the CZT.

Stress (low-dose) scans were acquired over $3 \mathrm{~min}$ and rest (high-dose) scans over $2 \mathrm{~min}$ as previously suggested (10). Images were reconstructed on the same workstation as was used for CT AC, with a new dedicated iterative algorithm with integrated collimator geometry modeling, using maximum penalized likelihood iterative reconstruction to obtain perfusion images in standard axes. The same operator, experienced in nuclear cardiology, reconstructed the datasets from both scanners to ascertain the use of identical reorientation parameters. Forty and 50 iterations for low and high counts, respectively, were performed, requiring approximately 1-min processing time. A Butterworth postprocessing filter (frequency, 0.37; order, 7) was applied to the reconstructed slices, according to the manufacturer's recommendations based on phantom studies.

\section{Quantitative Analysis}

Quantitative analysis was performed on MPI polar maps of noncorrected and attenuation-corrected images, and images were analyzed using a 20-segment left ventricular model for the left ventricle (14). Uptake was normalized to $100 \%$ of peak activity, and relative percentage count uptake of $\gamma$-ray emissions was assessed for each segment from standard SPECT camera and CZT data for low and high dose scans.

\section{Visual Analysis}

Masked visual analysis was performed for noncorrected and attenuation-corrected images with regard to the presence or absence of perfusion defects in the 3 main coronary territoriesthat is, left anterior descending artery, circumflex artery, and right coronary artery — as previously suggested (14). Two experienced nuclear cardiologists made the clinical analysis by consensus.

\section{Statistics}

SPSS software (15.0; SPSS Inc.) was used for statistical testing. Quantitative variables were expressed as mean \pm SD and categoric 


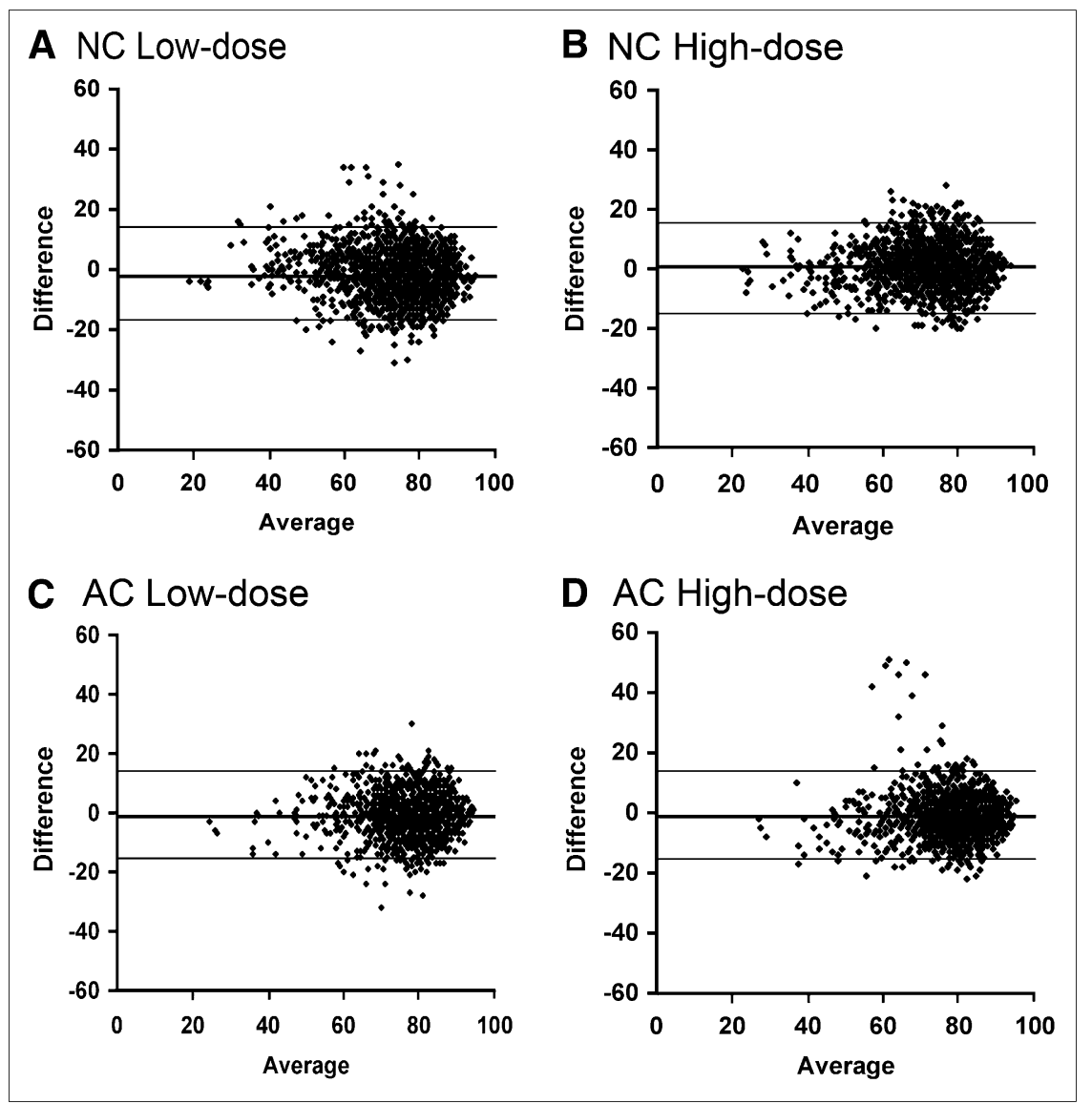

FIGURE 2. Bland-Altman limits of agreement for percentage segmental tracer uptake for noncorrected (NC) and attenuation-corrected (AC) myocardial perfusion images from CZT and standard SPECT cameras. variables as frequencies, mean, or percentages. Tracer uptake values (percentage of maximum myocardial uptake) from the CZT camera were compared segmentwise by intraclass correlation to the standard SPECT camera data, and Bland-Altman (BA) limits of agreement were calculated. Clinical agreement was assessed per coronary territory. $P$ values of less than 0.05 were considered statistically significant, and the $95 \%$ confidence intervals (CIs) are presented.

\section{RESULTS}

All 66 patients (48 men) successfully underwent MPI on the standard SPECT and CZT cameras. Forty patients $(61 \%)$ were referred because of suspected ischemia but unknown CAD and 26 patients (39\%) because of known CAD with suspected deterioration. Mean age $( \pm S D)$ was $65 \pm 11 \mathrm{y}$ (range, 38-82 y), and the mean body mass index was $27.2 \pm$ $3.7 \mathrm{~kg} / \mathrm{m}^{2}$ (range, $19.1-36.0 \mathrm{~kg} / \mathrm{m}^{2}$ ) for the men and $26.1 \pm$ $3,1 \mathrm{~kg} / \mathrm{m}^{2}$ (range, $21.7-30.8 \mathrm{~kg} / \mathrm{m}^{2}$ ) for the women. A mean $( \pm \mathrm{SD})$ of $335.7 \pm 25.0 \mathrm{MBq}$ of ${ }^{99 \mathrm{~m}} \mathrm{Tc}$-tetrofosmin (range, 303-455 MBq) for stress and $945.0 \pm 73.4 \mathrm{MBq}$ (range, 894.0-1,244 MBq) for rest were administered.

\section{Quantitative Analysis}

The intraclass correlation coefficient (between CZT and standard SPECT data) for quantitative tracer uptake of noncorrected images was 0.90 (95\% CI, 0.88-0.91; BA,
-18 to 15$)$ for low-dose images (stress) and 0.91 (95\% CI, 0.90-0.92; BA, -15 to 16) for high-dose images (rest). Similarly, for attenuation-corrected images, the correlation coefficient was 0.87 (95\% CI, $0.85-0.88$; BA, -16 to 14$)$ for low-dose images and 0.88 (95\% CI, 0.86-0.89; BA, -16 to 14 ) for high-dose images (Table 1; Fig. 2).

\section{Visual Analysis}

Image quality was diagnostic for all MPI on both the standard SPECT and the CZT cameras. Of the 198 possible coronary territories ( 66 patients $\times 3$ coronary territories), 61 revealed a perfusion defect at stress (low-dose scan) and 55 at rest (high-dose scan). For noncorrected images the clinical agreement per territory between the CZT and standard SPECT cameras was $96 \%$ for both stress (95\% CI, $0.92 \%-$

\section{TABLE 1. Percentage Segmental Tracer Uptake: Correlation Between CZT and Standard Cameras}

\begin{tabular}{|c|c|c|c|c|}
\hline \multirow[b]{2}{*}{ Dose } & \multicolumn{2}{|c|}{ NC } & \multicolumn{2}{|c|}{$A C$} \\
\hline & $r$ & $95 \% \mathrm{Cl}$ & $r$ & $95 \% \mathrm{Cl}$ \\
\hline Low & 0.90 & $0.88-0.91$ & 0.87 & $0.85-0.88$ \\
\hline High & 0.91 & $0.90-0.92$ & 0.88 & $0.86-0.89$ \\
\hline
\end{tabular}




\begin{tabular}{|c|c|c|c|c|c|c|c|c|c|}
\hline Per... & $\begin{array}{l}\text { True- } \\
\text { positive }\end{array}$ & $\begin{array}{l}\text { False- } \\
\text { positive }\end{array}$ & $\begin{array}{c}\text { True- } \\
\text { negative }\end{array}$ & $\begin{array}{c}\text { False- } \\
\text { negative }\end{array}$ & Sensitivity & Specificity & $\begin{array}{l}\text { Positive } \\
\text { predictive } \\
\text { value }\end{array}$ & $\begin{array}{l}\text { Negative } \\
\text { predictive } \\
\text { value }\end{array}$ & Agreement \\
\hline \multicolumn{10}{|c|}{ Patient $(n=66)$} \\
\hline $\begin{array}{l}\text { Low-dose } \\
\text { NC }\end{array}$ & 46 & 1 & 17 & 2 & 96 & 94 & 98 & 89 & 95 \\
\hline $\begin{array}{l}\text { Low-dose } \\
\text { AC }\end{array}$ & 24 & 1 & 40 & 1 & 96 & 98 & 96 & 98 & 97 \\
\hline $\begin{array}{l}\text { High-dose } \\
\text { NC }\end{array}$ & 44 & 2 & 18 & 2 & 96 & 90 & 96 & 90 & 94 \\
\hline $\begin{array}{l}\text { High-dose } \\
\text { AC }\end{array}$ & 21 & 1 & 44 & 0 & 100 & 98 & 100 & 95 & 98 \\
\hline \multicolumn{10}{|c|}{ Territory $(n=198)$} \\
\hline $\begin{array}{l}\text { Low-dose } \\
\text { NC }\end{array}$ & 56 & 3 & 134 & 5 & 92 & 98 & 95 & 96 & 96 \\
\hline $\begin{array}{l}\text { Low-dose } \\
\text { AC }\end{array}$ & 28 & 2 & 163 & 5 & 85 & 99 & 93 & 97 & 96 \\
\hline $\begin{array}{l}\text { High-dose } \\
\text { NC }\end{array}$ & 50 & 3 & 141 & 4 & 93 & 98 & 94 & 97 & 96 \\
\hline $\begin{array}{l}\text { High-dose } \\
\text { AC }\end{array}$ & 24 & 1 & 172 & 1 & 96 & 99 & 96 & 99 & 99 \\
\hline
\end{tabular}

$0.98 \%$ ) and rest (95\% CI, 0.93\%-0.99\%) scans. Similarly, for attenuation-corrected images the clinical agreement was $96 \%$ (95\% CI, 0.93\%-0.99\%) for stress scans and 99\% (95\% CI, $0.96 \%-0.99 \%$ ) for rest scans (Fig. 3). On the standard SPECT camera, 28 of 61 stress perfusion defects and 29 of 55 rest perfusion defects were unmasked as artifacts because they disappeared after correction with x-ray-based CT attenuation maps. These artifacts were predominantly located in the right coronary artery (stress, $n=24 / 28$; rest, $n=27 / 29$ ) and mainly found in male patients (stress, 22 men; rest, 25 men). The territory- and patient-based results are summarized in Table 2. Twenty-three of these 28 stress
FIGURE 3. Polar plot presentation of nuclear myocardial perfusion images from patient with normal perfusion (A) and from one with fixed anteroapical perfusion defect (B) with attenuation (AC) and without attenuation (NC) on standard SPECT camera (left) and novel CZT camera (right).
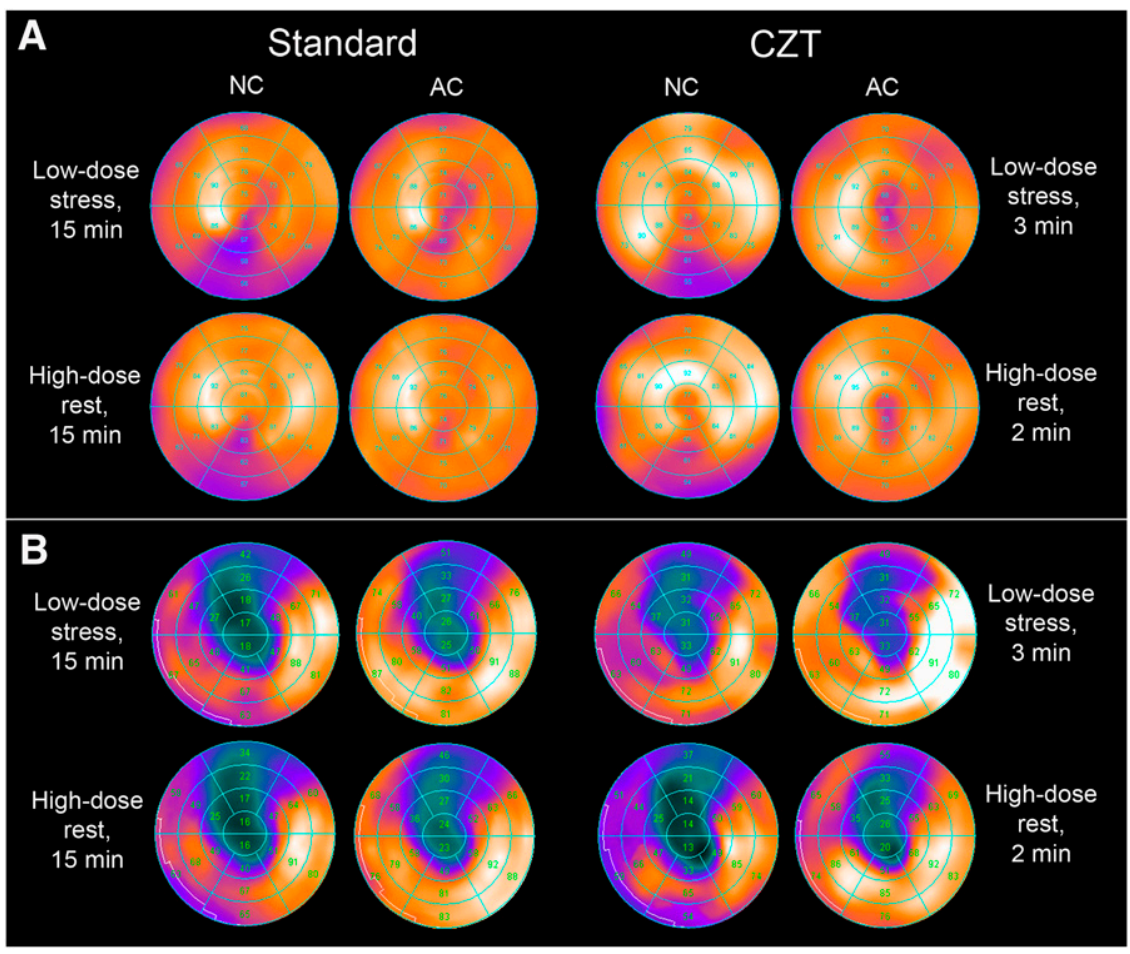
artifacts and 26 of these 29 rest artifacts were also present in CZT scans and were appropriately unmasked by AC.

\section{DISCUSSION}

Our study is the first, to our knowledge, to establish the feasibility and validate the accuracy of CT AC for MPI on the novel CZT camera, compared with scans from a conventional dual-head SPECT camera reconstructed with $\mathrm{AC}$ as a standard of reference. The CZT solid-state detector technology allows MPI with increased resolution and a minimized scan time $(10,17)$ and may therefore have the potential to emerge as an alternative to conventional SPECT cameras. Tissue attenuation plays a major role in SPECT MPI because it can affect the diagnostic accuracy, mainly by decreasing specificity, and as a consequence reduce costeffectiveness $(18,19)$ or even lead to nondiagnostic studies (20). Therefore, several strategies for AC have been evaluated, among which CT AC has proven most successful (1). Interestingly, the present study reveals that attenuation artifacts can nevertheless occur with this latest-generation camera, despite the increase of system sensitivity of CZT detectors and improved signal specificity of multipinhole technique. In the present study with 66 patients and 198 possible coronary territories, 28 defects at stress and 29 defects at rest were unmasked as attenuation artifacts in MPI on a standard SPECT camera. These artifacts were predominantly located in the right coronary artery territory, in line with several previous reports $(1,13,21)$. All defects found in conventional SPECT MPI and classified as artifacts by CT AC were also identified in CZT MPI.

We have chosen CT AC because this method has been reported to be technically well established not only in hybrid SPECT/CT scanners integrating a $\gamma$-camera with a CT device but also when CT AC maps are obtained from a standalone CT scanner (13).

CT AC has been reported in 1 study to substantially increase confidence and improve diagnostic performance, primarily by increasing the specificity of MPI (21). The degree of specificity improvement in that study depended on the interpretative attitude, as readers prone to high sensitivity or with less experience had the greatest gain in the normalcy rate, whereas readers prone to higher specificity had improvement in sensitivity and specificity but not the normalcy rate. Thus, improvements in any of the diagnostic variables were not counterbalanced by degradation of other variables, supporting that CT-based AC of SPECT MPI is well suited for routine use in clinical practice. The unenhanced low-dose CT scan for AC has been shown to allow the calculation of the calcium score (13), which may improve the diagnostic and prognostic accuracy of MPI when used as an adjunct to SPECT MPI $(22,23)$. The new CZT camera differs in many respects from conventional SPECT $\gamma$-cameras, because not only a new semiconductor to replace the $\mathrm{NaI}$ crystals but also multiple detector heads allowing simultaneous image acquisition using a pinhole geometry in 19 angles have been introduced.
Despite these significant differences, x-ray-based CT AC remains feasible and helpful for the successful discrimination of artifacts from true perfusion defects. In fact, these results show excellent agreement with conventional SPECT MPI scans for both quantitative segmental tracer uptake and clinical findings and diagnostic conclusions.

The following limitations have to be considered: we included patients with a body mass index ranging from 19.1 to $36.0 \mathrm{~kg} / \mathrm{m}^{2}$ into our study population. Therefore, our results must be read with caution when extrapolating them to patients with a substantially different body mass index, particularly when exceeding the upper range. Similarly, as the present results are mainly based on inferior artifacts (generally perceived as male pattern) because of underrepresentation of women in this study (27\%), an evaluation in a large population of female patients should be considered. Furthermore, we did not use invasive coronary angiography as a standard of reference. Thus, we theoretically cannot entirely exclude overcorrection of true inferior artifacts.

However, we felt it more appropriate to use an MPI modality as a standard of reference for a study assessing MPI with a new device, particularly because the accuracy of CT AC for SPECT has been well established (21).

\section{CONCLUSION}

Our results support the feasibility of AC of MPI on the novel CZT camera. Segmental tracer uptake correlated strongly with AC of MPI on a conventional SPECT camera, and clinical agreement was excellent.

\section{ACKNOWLEDGMENTS}

We thank our radiography team, especially Edlira Loga and Ennio Müller, for their excellent technical support. The study was supported by a grant from the Swiss National Science Foundation (SNSF) and by the ZIHP (Zurich Center for Integrative Human Physiology, University of Zurich, Switzerland). The university hospital holds a research contract with GE Healthcare.

\section{REFERENCES}

1. Fricke E, Fricke H, Weise R, et al. Attenuation correction of myocardial SPECT perfusion images with low-dose CT: evaluation of the method by comparison with perfusion PET. J Nucl Med. 2005;46:736-744.

2. Tsui BM, Gullberg GT, Edgerton ER, et al. Correction of nonuniform attenuation in cardiac SPECT imaging. J Nucl Med. 1989;30:497-507.

3. Nishina H, Slomka PJ, Abidov A, et al. Combined supine and prone quantitative myocardial perfusion SPECT: method development and clinical validation in patients with no known coronary artery disease. J Nucl Med. 2006;47:51-58.

4. Tan P, Bailey DL, Meikle SR, Eberl S, Fulton RR, Hutton BF. A scanning line source for simultaneous emission and transmission measurements in SPECT. J Nucl Med. 1993;34:1752-1760.

5. Bocher M, Balan A, Krausz Y, et al. Gamma camera-mounted anatomical X-ray tomography: technology, system characteristics and first images. Eur J Nucl Med. 2000;27:619-627.

6. Lang TF, Hasegawa BH, Liew SC, et al. Description of a prototype emissiontransmission computed tomography imaging system. J Nucl Med. 1992;33:18811887.

7. Malkerneker D, Brenner R, Martin WH, et al. CT-based attenuation correction versus prone imaging to decrease equivocal interpretations of rest/stress Tc- $99 \mathrm{~m}$ tetrofosmin SPECT MPI. J Nucl Cardiol. 2007;14:314-323. 
8. Koepfli P, Hany TF, Wyss CA, et al. CT attenuation correction for myocardial perfusion quantification using a PET/CT hybrid scanner. J Nucl Med. 2004;45: $537-542$.

9. O’Connor MK, Kemp BJ. Single-photon emission computed tomography/ computed tomography: basic instrumentation and innovations. Semin Nucl Med. 2006;36:258-266.

10. Herzog BA, Buechel RR, Katz R, et al. Nuclear myocardial perfusion imaging with a cadmium-zinc-telluride detector technique: optimized protocol for scan time reduction. J Nucl Med. 2009;51:46-51.

11. Buechel RR, Herzog BA, Husmann L, et al. Ultrafast nuclear myocardial perfusion imaging on a new gamma camera with semiconductor detector technique: first clinical validation. Eur J Nucl Med Mol Imaging. 2010;37:773-778.

12. Hesse B, Tagil K, Cuocolo A, et al. EANM/ESC procedural guidelines for myocardial perfusion imaging in nuclear cardiology. Eur J Nucl Med Mol Imaging. 2005;32:855-897.

13. Schepis T, Gaemperli O, Koepfli P, et al. Use of coronary calcium score scans from stand-alone multislice computed tomography for attenuation correction of myocardial perfusion SPECT. Eur J Nucl Med Mol Imaging. 2007;34:11-19.

14. Hansen CL, Goldstein RA, Akinboboye OO, et al. Myocardial perfusion and function: single photon emission computed tomography. J Nucl Cardiol. 2007;14:e39-e60.

15. Blevis I, Tsukerman L, Volokh L, Hugg J, Jansen F, Bouhnik J. CZT gamma camera with pinhole collimator: spectral measurements. IEEE Nucl Sci Symp Conf Rec. 2008:4931-4932.
16. Madsen MT. Recent advances in SPECT imaging. J Nucl Med. 2007;48:661673.

17. Esteves FP, Raggi P, Folks RD, et al. Novel solid-state-detector dedicated cardiac camera for fast myocardial perfusion imaging: multicenter comparison with standard dual detector cameras. J Nucl Cardiol. 2009;16:927-934.

18. Fleischmann KE, Hunink MG, Kuntz KM, Douglas PS. Exercise echocardiography or exercise SPECT imaging? A meta-analysis of diagnostic test performance. JAMA. 1998;280:913-920.

19. Kuntz KM, Fleischmann KE, Hunink MG, Douglas PS. Cost-effectiveness of diagnostic strategies for patients with chest pain. Ann Intern Med. 1999;130: 709-718.

20. McQuaid SJ, Hutton BF. Sources of attenuation-correction artefacts in cardiac PET/CT and SPECT/CT. Eur J Nucl Med Mol Imaging. 2008;35:1117-1123.

21. Masood Y, Liu YH, Depuey G, et al. Clinical validation of SPECT attenuation correction using $\mathrm{X}$-ray computed tomography-derived attenuation maps: multicenter clinical trial with angiographic correlation. J Nucl Cardiol. 2005; 12:676-686.

22. Schepis T, Gaemperli O, Koepfli P, et al. Added value of coronary artery calcium score as an adjunct to gated SPECT for the evaluation of coronary artery disease in an intermediate-risk population. $J$ Nucl Med. 2007;48:1424-1430.

23. Berman DS, Wong ND, Gransar H, et al. Relationship between stress-induced myocardial ischemia and atherosclerosis measured by coronary calcium tomography. J Am Coll Cardiol. 2004;44:923-930. 